\title{
Measurements of Discrete Symmetries in the Neutral Kaon System with the CPLEAR (PS195) Experiment
}

\author{
Thomas Ruf \\ CERN, CH-1211, Geneva 23, Switzerland \\ thomas.ruf@cern.ch
}

\begin{abstract}
The antiproton storage ring LEAR offered unique opportunities to study the symmetries which exist between matter and antimatter. At variance with other approaches at this facility, CPLEAR was an experiment devoted to the study of $\mathcal{T}, \mathcal{C P} \mathcal{T}$ and $\mathcal{C P}$ symmetries in the neutral kaon system. It measured with high precision the time evolution of initially strangeness-tagged $\mathrm{K}^{0}$ and $\overline{\mathrm{K}}^{0}$ states to determine the size of violations with respect to these symmetries in the context of a systematic study. In parallel, limits concerning quantum-mechanical predictions (EPR paradox, coherence of the wave function) or the equivalence principle of general relativity have been obtained. This article will first discuss briefly the unique low energy antiproton storage ring LEAR followed by a description of the CPLEAR experiment, including the basic formalism necessary to understand the time evolution of a neutral kaon state and the main results related to measurements of discrete symmetries in the neutral kaon system. An excellent and exhaustive review of the CPLEAR experiment and all its measurements is given in Ref. 1.
\end{abstract}

\section{The Low Energy Antiproton Ring}

The Low Energy Antiproton Ring (LEAR) $)^{2,3}$ decelerated and stored antiprotons for eventual extraction to the experiments located in the South Hall. It was built in 1982 and operated until 1996, when it was converted into the Low Energy Ion Ring (LEIR), which provides lead-ion injection for the Large Hadron Collider (LHC). Under the LEAR programme, four machines - the Proton Synchrotron (PS), the Antiproton Collector (AC), the Antiproton Accumulator (AA), and LEAR worked together to collect, cool and decelerate antiprotons for use in experiments. Protons accelerated to $26 \mathrm{GeV} / c$ by the PS created antiprotons in collisions with a fixed target. A magnetic spectrometer selected the emerging antiprotons $(3.6 \mathrm{GeV} / c)$ and injected them into the AC. Here they stayed for $4.8 \mathrm{~s}$ to reduce their momentum spread by means of stochastic cooling before being stored for a long time in the AA. Whenever the LEAR machine was ready to take a shot $\left(\approx 5 \times 10^{9}\right)$ of $\overline{\mathrm{p}}$, the AA released a part of its stack to the PS, where the $\overline{\mathrm{p}}$ 's were decelerated to $609 \mathrm{MeV} / c$, injected into LEAR, and stochastically cooled down for another $5 \mathrm{~min}$ to a momentum spread of $\sigma_{p} / p=10^{-3}$. This was followed by electron cooling, resulting in a relative momentum spread of only $5 \times 10^{-4}$. LEAR had been equipped 
with fast and ultra-slow extraction systems, the latter being used for the CPLEAR experiment providing a rate of $1 \mathrm{MHz}$ antiprotons in spills of about $1 \mathrm{~h}$. The last part of extraction line comprised two horizontal and two vertical bending magnets followed by a quadrupole doublet to align and focus the beam on the target in the centre of the detector. The size of the beam spot on the target had a FWHM of about $3 \mathrm{~mm}$.

\section{The CPLEAR Experimental Method}

CPLEAR made use of charge-conjugate particles $\mathrm{K}^{0}$ and $\overline{\mathrm{K}}^{0}$ produced in $\mathrm{p} \overline{\mathrm{p}}$ collisions at rest, with a flavour of strangeness different for particles $\left(\mathrm{K}^{0}\right)$ and antiparticles $\left(\overline{\mathrm{K}}^{0}\right)$ :

$$
\overline{\mathrm{p} p} \rightarrow \begin{aligned}
& \mathrm{K}^{-} \pi^{+} \mathrm{K}^{0} \\
& \mathrm{~K}^{+} \pi^{-} \overline{\mathrm{K}}^{0}
\end{aligned} .
$$

The conservation of strangeness in the strong interaction dictates that a $\mathrm{K}^{0}$ is accompanied by a $\mathrm{K}^{-}$, and a $\overline{\mathrm{K}}^{0}$ by a $\mathrm{K}^{+}$. Hence, the strangeness of the neutral kaon at production was tagged by measuring the charge sign of the accompanying charged kaon and was therefore known event by event. If the neutral kaon subsequently decayed to e $\pi \nu$, its strangeness could also be tagged at the decay time by the charge of the decay electron. Indeed, in the limit that only transitions with $\Delta S=\Delta Q$ take place, neutral kaons decay to $\mathrm{e}^{+}$if the strangeness is positive at the decay time and to $\mathrm{e}^{-}$if it is negative. For each initial strangeness, the number of neutral kaon decays was measured as a function of the decay time $\tau$. These numbers, $N_{f}(\tau)$ and $\bar{N}_{f}(\tau)$ for a non-leptonic final state $f$, or $N_{ \pm}(\tau)$ and $\bar{N}_{\mp}(\tau)$ for an e $\pi \nu$ final state, were combined to form asymmetries, thus dealing mainly with ratios between measured quantities. However, the translation of measured numbers of events into decay rates requires acceptance factors which do not cancel in such asymmetries (a), residual background (b), and regeneration effects (c) to be taken into account.

(a) The major effect arises from the strangeness tagging of the neutral kaon state with the help of detecting and identifying the charge of the accompanying $\mathrm{K}^{\mp} \pi^{ \pm}$track-pair at the production vertex, and at the decay vertex with the $\mathrm{e}^{\mp} \pi^{ \pm}$track-pair. Small misalignments of detector components result in different momentum dependent efficiencies for reconstructing positively and negatively charged particles. This effect can be mitigated by changing frequently the polarity of the solenoid magnet, few times per day. A second charge dependent effect arises from different interaction probabilities of particles and antiparticles with the detector material made of matter and not of antimatter. These differences are determined in bins of the kinematics phasespace with large statistics samples of $\mathrm{K}^{0} \rightarrow \pi^{+} \pi^{-}$at short decay times for the accompanying $\mathrm{K}^{\mp} \pi^{ \pm}$track-pair, and with calibration data for the $\mathrm{e}^{\mp} \pi^{ \pm}$ track-pair obtained in a beam at the Paul-Scherrer-Institute (PSI) cyclotron. 
(b) The background events mainly consist of neutral kaon decays to final states other than the signal. Since to a high degree of accuracy the amount of background is the same for initial $\mathrm{K}^{0}$ and $\overline{\mathrm{K}}^{0}$ the contribution cancels in the numerator but not in the denominator of any asymmetry: thus diluting any asymmetry. Their contributions are obtained by Monte Carlo simulations and taken into account by the fits to the asymmetries.

(c) The regeneration probabilities of $\mathrm{K}^{0}$ and $\overline{\mathrm{K}}^{0}$ propagating through the detector material are not the same, thus making the measured ratio of initial $\overline{\mathrm{K}}^{0}$ and $\mathrm{K}^{0}$ decay events at time $\tau$ different from that expected in vacuum. The effect is called regeneration, since it also leads to the creation of $\mathrm{K}_{\mathrm{S}}$ particles when a beam of $\mathrm{K}_{\mathrm{L}}$ particles propagates through material, which does not happen in vacuum. A dedicated experimental setup had been used to improve the knowledge on regeneration amplitudes, magnitudes and phases, in the momentum range relevant for the CPLEAR experiment. ${ }^{4}$ The effect is being corrected for by applying a weight to each $\mathrm{K}^{0}\left(\overline{\mathrm{K}}^{0}\right)$ event equal to the ratio of the decay probabilities for an initial $\mathrm{K}^{0}\left(\overline{\mathrm{K}}^{0}\right)$ propagating in vacuum and through the detector.

\section{The CPLEAR Detector}

The detector specifications were based on the following essential experimental requirements:

- A very efficient charged kaon identification to separate the signal from the (very) large number of multi-pion annihilation channels.

- To distinguish between the various neutral kaon decay channels.

- To measure the decay proper time between 0 and $\approx 20 \mathrm{~K}_{\mathrm{S}}$ mean lives. At the highest $\mathrm{K}^{0}$ momentum measured in our experiment $(750 \mathrm{MeV} / c)$, the $\mathrm{K}_{\mathrm{S}}$ mean decay length is $4 \mathrm{~cm}$. This sets the size of the cylindrical decay volume to a radius of $\approx 60 \mathrm{~cm}$.

- To minimise material to keep the regeneration corrections small, resulting for example in the use of a pressurised hydrogen target instead of liquid hydrogen target.

- To acquire a large number of events, which required both a high rate sophisticated trigger and data acquisition system (1 MHz annihilation rate) and a large geometrical coverage.

Since the antiprotons annihilate at rest, the particles are produced isotropically, thus the detector had a typical near- $4 \pi$ geometry. The whole detector was embedded in a (3.6 m long, $2 \mathrm{~m}$ diameter) warm solenoidal magnet which provided a $0.44 \mathrm{~T}$ uniform field. The general layout of the CPLEAR experiment is shown in Fig. 1; a comprehensive description of the detector is found in Ref. 5. The incoming 


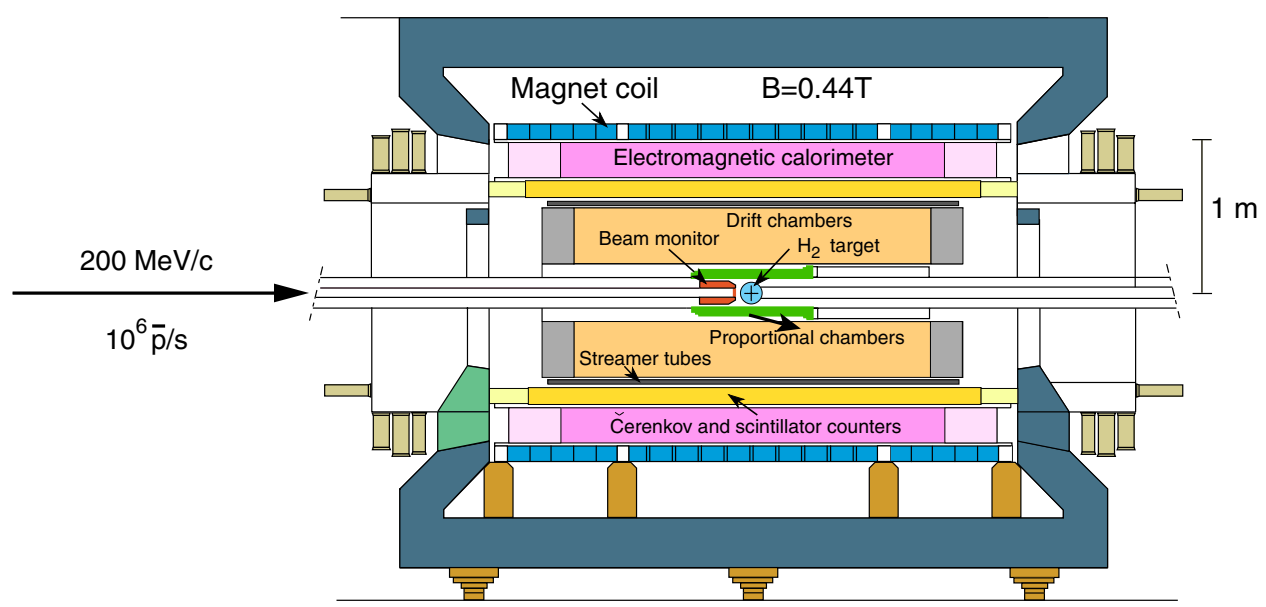

(a)

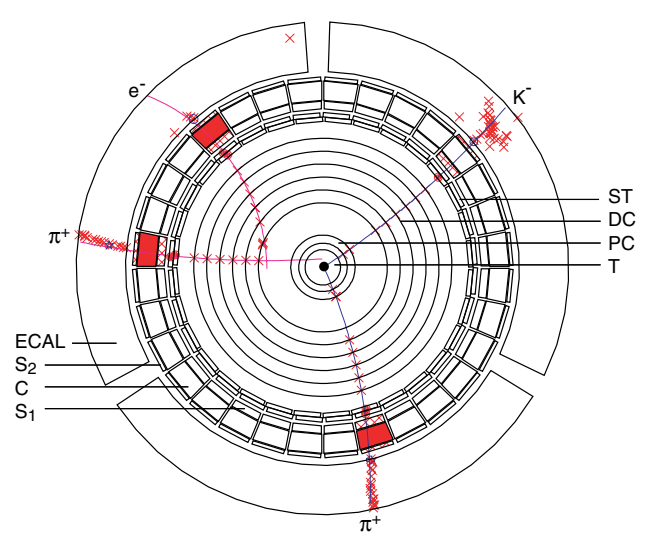

(b)

Fig. 1. CPLEAR detector: (a) longitudinal view and (b) transverse view and display of an event, $\overline{\mathrm{p}} \mathrm{p}$ (not shown) $\rightarrow \mathrm{K}^{-} \pi^{+} \mathrm{K}^{0}$ with the neutral kaon decaying to $\mathrm{e}^{-} \pi^{+} \bar{\nu}$. The view (b) is magnified twice with respect to (a) and does not show the magnet coils and outer detector components. In both views the central region refers to the early data taking without PC0.

antiprotons were stopped in a pressurised hydrogen gas target. For data taken up to mid-1994 the target was a sphere of $7 \mathrm{~cm}$ radius at 16 bar pressure. After that date it was replaced by a $1.1 \mathrm{~cm}$ radius cylindrical target at 27 bar pressure. A series of light-weighted cylindrical tracking detectors provided information about the trajectories of charged particles in order to determine their charge signs, momenta and positions. There were two proportional chambers $(9.5$ and $12.7 \mathrm{~cm}$ in radius, measuring $r \Phi$ ), six drift chambers (from 25 to $60 \mathrm{~cm}$, measuring $r \Phi, z$ ) and two layers of streamer tubes (for a fast $z$ determination within $600 \mathrm{~ns}$ ). The total material in the target and tracking chambers amounted to $\approx 1 \%$ equivalent radiation length $\left(X_{0}\right)$. After trackfit, the spatial resolution was better than $350 \mu \mathrm{m}$ in $r$ and 
$r \Phi$, and $2 \mathrm{~mm}$ in $z$ with sufficient good momentum resolution $(\Delta p / p$ between $5 \%$ and $10 \%$ ).

The tracking detectors were followed by the particle-identification detector (PID), which carried out the charged-kaon identification. The PID comprised a threshold Cherenkov detector, which was mainly effective for $K / \pi$ separation above $350 \mathrm{MeV} / c$ momentum, and scintillators which measured the energy loss $(d E / d x)$ and the time of flight of charged particles. The PID recognised in $\approx 60 \mathrm{~ns}$ the presence of a charged kaon out of a background 250 times higher. The Cherenkov threshold was $300 \mathrm{MeV} / c$ for pions and $700 \mathrm{MeV} / c$ for kaons. The PID was also used to separate electrons from pions below $350 \mathrm{MeV} / c$.

The outermost detector was a lead/gas sampling calorimeter (ECAL) used to detect the photons produced in $\pi^{0}$ decays. It consisted of 18 layers of $1.5 \mathrm{~mm}$ lead converters and high-gain tubes, the latter sandwiched between two layers of pick-up strips $\left( \pm 30^{\circ}\right.$ with respect to the tubes), for a total of 64000 readout channels. The design criteria of the calorimeter were mainly dictated by the required accuracy on the reconstruction of the $\mathrm{K}^{0} \rightarrow 2 \pi^{0}$ or $3 \pi^{0}$ decay vertices. The calorimeter provided e/ $\pi$ separation at higher momenta $(p>300 \mathrm{MeV} / c)$ complementary to the PID.

The high annihilation rate and the small value of the branching ratio for the signal reaction $\left(\approx 2 \times 10^{-3}\right)$ made it necessary to develop a sophisticated trigger and data acquisition system to limit the amount of recorded data and to minimise the dead-time of the experiment. A set of hardwired processors (HWP) was specially designed to reject unwanted events fast and efficient, by providing a full event reconstruction in a few microseconds. The decisions were based on fast recognition of the charged kaon (using the PID hit maps), the number and topology of the charged tracks, the particle identification (using energy-loss, time-of-flight and Cherenkov light response) and kinematic constraints, as well as the number of showers in the ECAL. The overall rejection factor of the trigger was about $10^{3}$, allowing a read-out rate of $\approx 450$ events per second at an average beam rate of $800 \mathrm{kHz}$.

In order to control the bias introduced by the trigger selections, it was essential to confirm the primary $\mathrm{K} \pi$ pairs found by the trigger with the primary $\mathrm{K} \pi$ pairs found by the offline reconstruction. This matching procedure was achieved by running the trigger simulation on the selected events, requiring the event to pass the trigger criteria with which the data were written, and rejecting events where the trigger and offline reconstruction disagreed on primary tracks. In addition, minimum-bias data, requiring only the coincidence between an incoming $\overline{\mathrm{p}}$ signal and a signal in one of the scintillators, were collected at least three times a day, thus providing a representative set of the overall data to be used for calibration purposes and trigger studies.

The CPLEAR detector was fully operational between 1992 and 1996, collecting a total number of antiprotons equal to $1.1 \times 10^{13}$. The recorded data of 12 Tbytes consisted of nearly $2 \times 10^{8}$ decays of strangeness-tagged neutral kaons, of which 
$7 \times 10^{7}$ decays are to $\pi^{+} \pi^{-}$with a decay time greater than $1 \tau_{\mathrm{S}}, 1.3 \times 10^{6}$ to $\mathrm{e} \pi \nu$, $2 \times 10^{6}$ to $\pi^{0} \pi^{0}, 5 \times 10^{5}$ to $\pi^{+} \pi^{-} \pi^{0}$, and $1.7 \times 10^{4}$ to $\pi^{0} \pi^{0} \pi^{0}$. With these data CPLEAR achieved a number of results on the discrete symmetries in the neutral kaon system, ${ }^{6-12}$ and measured other related quantities. ${ }^{4,}{ }^{13-15}$ The large statistics of decays to $\pi^{+} \pi^{-}$allowed testing the equivalence principle of general relativity, ${ }^{16}$ by looking at possible annual, monthly and diurnal modulations of the $\mathcal{C P}$ violation parameter $\eta_{+-}$caused by variations in astrophysical potentials. With a slightly modified setup originally introduced to measure neutral kaon forward scattering cross-sections in carbon, ${ }^{17}$ CPLEAR was also able to perform an Einstein-PodolskiRosen-type experiment. ${ }^{18}$ Combining several CPLEAR measurements enabled tests of quantum mechanics, ${ }^{19}$ setting limits on parameters describing the possible evolution of pure states into mixed states, sensitive to physics at ultra-high energies, as well as precise determinations of mass and lifetime differences between $\mathrm{K}^{0}$ and its antiparticle $\overline{\mathrm{K}}^{0}$ using the unitarity relation. ${ }^{20,21}$ Some of these measurements will be discussed in more detail in the following sections after a short introduction into the formalism of the time evolution of neutral kaon states.

\section{Phenomenology of the Neutral Kaon System}

\subsection{Time evolution}

In the absence of any strangeness-violating interaction, the stationary states $\left|\mathrm{K}^{0}\right\rangle$ and $\left|\overline{\mathrm{K}}^{0}\right\rangle$ of a $\mathrm{K}^{0}$-meson and $\overline{\mathrm{K}}^{0}$-meson respectively, are mass eigenstates of the strong and electromagnetic interactions and of strangeness $S$ :

$$
\begin{aligned}
\left(\mathcal{H}_{\mathrm{st}}+\mathcal{H}_{\mathrm{em}}\right)\left|\mathrm{K}^{0}\right\rangle=m_{0}\left|\mathrm{~K}^{0}\right\rangle & \left(\mathcal{H}_{\mathrm{st}}+\mathcal{H}_{\mathrm{em}}\right)\left|\overline{\mathrm{K}}^{0}\right\rangle=m_{0}\left|\overline{\mathrm{K}}^{0}\right\rangle, \\
S\left|\mathrm{~K}^{0}\right\rangle=+\left|\mathrm{K}^{0}\right\rangle & S\left|\overline{\mathrm{K}}^{0}\right\rangle=-\left|\overline{\mathrm{K}}^{0}\right\rangle .
\end{aligned}
$$

Since the strangeness-violating interaction $\mathcal{H}_{\mathrm{wk}}$ is much weaker than the strong and electromagnetic interaction, perturbation theory can be applied (Wigner-Weisskopf approach). ${ }^{22,23}$ The time evolution of the neutral kaon wave function is then described by the following differential equation: ${ }^{24,} 25$

$$
i \frac{\mathrm{d}}{\mathrm{d} \tau}\left(\begin{array}{l}
\mathrm{K}^{0}(\tau) \\
\overline{\mathrm{K}}^{0}(\tau)
\end{array}\right)=\Lambda\left(\begin{array}{l}
\mathrm{K}^{0}(\tau) \\
\overline{\mathrm{K}}^{0}(\tau)
\end{array}\right)=\left(M-\frac{i}{2} \Gamma\right)\left(\begin{array}{l}
\mathrm{K}^{0}(\tau) \\
\overline{\mathrm{K}}^{0}(\tau)
\end{array}\right),
$$

where $\Lambda$ can be split into two Hermitian matrices $M$ and $\Gamma$, called mass and decay matrices respectively. The matrix elements of $\Lambda$ are given by:

$$
\begin{aligned}
\Lambda_{i j}= & m_{0} \delta_{i j}+\left\langle i\left|\mathcal{H}_{\mathrm{wk}}\right| j\right\rangle+\sum_{f} \mathcal{P}\left(\frac{\left\langle i\left|\mathcal{H}_{\mathrm{wk}}\right| f\right\rangle\left\langle f\left|\mathcal{H}_{\mathrm{wk}}\right| j\right\rangle}{m_{0}-E_{f}}\right) \\
& -i \pi \sum_{f}\left\langle i\left|\mathcal{H}_{\mathrm{wk}}\right| f\right\rangle\left\langle f\left|\mathcal{H}_{\mathrm{wk}}\right| j\right\rangle \delta\left(m_{0}-E_{f}\right),
\end{aligned}
$$

where $\mathcal{P}$ stands for the principal part and the indices $i, j=1$ and $i, j=2$ correspond to $\mathrm{K}^{0}$ and $\overline{\mathrm{K}}^{0}$ respectively. They can be calculated within the Standard Model, 
although with large uncertainties because of non-perturbative effects. The same formalism applies to the two neutral $B$-meson systems $\left(B_{d}\right.$ and $\left.B_{s}\right)$ where the matrix elements of $\Lambda$ can be calculated rather reliable due to the much larger mass of the $b$-quark compared to the $s$-quark. Since no direct $K^{0}-\overline{\mathrm{K}}^{0}$ transition exists within the Standard Model, the second term of Eq. (5) vanishes. We use the following parametrisation of $\Lambda$ with eight real and positive parameters:

$$
\Lambda=\left(\begin{array}{cc}
m_{\mathrm{K}^{0}} & M_{12} \mathrm{e}^{\mathrm{i} \varphi_{\mathrm{M}}} \\
M_{12} \mathrm{e}^{-\mathrm{i} \varphi_{\mathrm{M}}} & m_{\overline{\mathrm{K}}^{0}}
\end{array}\right)-\frac{i}{2}\left(\begin{array}{cc}
\Gamma_{\mathrm{K}^{0}} & \Gamma_{12} \mathrm{e}^{\mathrm{i} \varphi_{\Gamma}} \\
\Gamma_{12} \mathrm{e}^{-\mathrm{i} \varphi_{\Gamma}} & \Gamma_{\overline{\mathrm{K}}^{0}}
\end{array}\right),
$$

where $m_{\mathrm{K}^{0}}$ and $m_{\overline{\mathrm{K}}^{0}}$ are equal to the masses, and $1 / \Gamma_{\mathrm{K}^{0}}$ and $1 / \Gamma_{\overline{\mathrm{K}}^{0}}$ to the lifetimes of the $\mathrm{K}^{0}$ and $\overline{\mathrm{K}}^{0}$ states respectively.

The time evolution of initially pure $\mathrm{K}^{0}$ and $\overline{\mathrm{K}}^{0}$ states is given by

$$
\left(\begin{array}{l}
\mathrm{K}^{0}(\tau) \\
\overline{\mathrm{K}}^{0}(\tau)
\end{array}\right)=T(\tau)\left(\begin{array}{l}
\mathrm{K}^{0}(0) \\
\overline{\mathrm{K}}^{0}(0)
\end{array}\right)
$$

with

$$
\begin{aligned}
T(\tau) & =\left(\begin{array}{c}
f_{+}(\tau)+\frac{\Lambda_{22}-\Lambda_{11}}{\Delta \lambda} f_{-}(\tau)-2 \frac{\Lambda_{21}}{\Delta \lambda} f_{-}(\tau), \\
f_{+}(\tau)-\frac{\Lambda_{22}-\Lambda_{11}}{\Delta \lambda} f_{-}(\tau)-2 \frac{\Lambda_{12}}{\Delta \lambda} f_{-}(\tau)
\end{array}\right) \\
f_{ \pm}(\tau) & =\frac{\mathrm{e}^{-i \lambda_{\mathrm{S}} \tau} \pm \mathrm{e}^{-i \lambda_{\mathrm{L}} \tau}}{2}, \\
\lambda_{L, S} & =m_{L, S}-\frac{i}{2} \Gamma_{L, S}=\frac{\Lambda_{11}+\Lambda_{22}}{2} \pm \sqrt{\frac{\left(\Lambda_{22}-\Lambda_{11}\right)^{2}}{4}+\Lambda_{12} \Lambda_{21}}, \\
\Delta \lambda & =\lambda_{\mathrm{L}}-\lambda_{\mathrm{S}}=\sqrt{\left(\Lambda_{22}-\Lambda_{11}\right)^{2}+4 \Lambda_{12} \Lambda_{21}},
\end{aligned}
$$

where $\lambda_{\mathrm{S}}$ and $\lambda_{\mathrm{L}}$ are the eigenvalues of the matrix $\Lambda$. The corresponding eigenvectors are given by:

$$
\begin{aligned}
& \left|\mathrm{K}_{\mathrm{S}}\right\rangle=\frac{\mathrm{e}^{i \varphi_{\mathrm{S}}}}{\sqrt{1+\left|r_{\mathrm{S}}\right|^{2}}}\left(r_{\mathrm{S}}\left|\mathrm{K}^{0}\right\rangle+\left|\overline{\mathrm{K}}^{0}\right\rangle\right), \\
& \left|\mathrm{K}_{\mathrm{L}}\right\rangle=\frac{\mathrm{e}^{i \varphi_{\mathrm{L}}}}{\sqrt{1+\left|r_{\mathrm{L}}\right|^{2}}}\left(r_{\mathrm{L}}\left|\mathrm{K}^{0}\right\rangle+\left|\overline{\mathrm{K}}^{0}\right\rangle\right)
\end{aligned}
$$

with arbitrary phases $\varphi_{\mathrm{S}}, \varphi_{\mathrm{L}}$ and

$$
\begin{aligned}
& r_{\mathrm{S}}=\frac{2 M_{12}}{\Lambda_{22}-\Lambda_{11}-\Delta \lambda} \\
& r_{\mathrm{L}}=\frac{2 M_{12}}{\Lambda_{22}-\Lambda_{11}+\Delta \lambda}
\end{aligned}
$$




\section{2. $\quad$ Discrete symmetries}

$\mathcal{C P}$ and $\mathcal{C P} \mathcal{T}$ transformations change a stationary $\mathrm{K}^{0}$ state into a $\overline{\mathrm{K}}^{0}$ state and vice versa, whereas a $\mathcal{T}$ transformation does not alter the states except for an arbitrary phase:

$$
\begin{aligned}
& \mathcal{C P}\left|\mathrm{K}^{0}\right\rangle=\quad \mathrm{e}^{\mathrm{i} \phi_{\mathrm{CP}}}\left|\overline{\mathrm{K}}^{0}\right\rangle, \quad \mathcal{C} \mathcal{P}\left|\overline{\mathrm{K}}^{0}\right\rangle=\quad \mathrm{e}^{-\mathrm{i} \phi_{\mathrm{CP}}}\left|\mathrm{K}^{0}\right\rangle, \\
& \mathcal{T}\left|\mathrm{K}^{0}\right\rangle=\quad \mathrm{e}^{\mathrm{i} \phi_{\mathrm{T}}}\left|\mathrm{K}^{0}\right\rangle, \quad \mathcal{T}\left|\overline{\mathrm{K}}^{0}\right\rangle=\quad \mathrm{e}^{\mathrm{i} \bar{\phi}_{\mathrm{T}}}\left|\overline{\mathrm{K}}^{0}\right\rangle, \\
& \mathcal{C P} \mathcal{T}\left|\mathrm{K}^{0}\right\rangle=\mathrm{e}^{\mathrm{i}\left(\phi_{\mathrm{CP}}+\phi_{\mathrm{T}}\right)}\left|\overline{\mathrm{K}}^{0}\right\rangle, \mathcal{C} \mathcal{P} \mathcal{T}\left|\overline{\mathrm{K}}^{0}\right\rangle=\mathrm{e}^{\mathrm{i}\left(-\phi_{\mathrm{CP}}+\bar{\phi}_{\mathrm{T}}\right)}\left|\mathrm{K}^{0}\right\rangle .
\end{aligned}
$$

By requiring $\mathcal{C P} \mathcal{T}\left|\mathrm{K}^{0}\right\rangle=\mathcal{T} \mathcal{C P}\left|\mathrm{K}^{0}\right\rangle$, it follows for the phases $\phi_{i}$ that

$$
2 \phi_{\mathrm{CP}}=\bar{\phi}_{\mathrm{T}}-\phi_{\mathrm{T}} \text {. }
$$

If $\Lambda$ is invariant under $\mathcal{T}, \mathcal{C P} \mathcal{T}$ or $\mathcal{C P}$ transformations, the following conditions must be satisfied:

$$
\begin{aligned}
& \mathcal{T} \quad:\left|\Lambda_{12}\right|=\left|\Lambda_{21}\right|, \\
& \mathcal{C P} \mathcal{T}: \Lambda_{11}=\Lambda_{22}, \\
& \mathcal{C P} \quad:\left|\Lambda_{12}\right|=\left|\Lambda_{21}\right| \quad \text { and } \quad \Lambda_{11}=\Lambda_{22} .
\end{aligned}
$$

It is convenient to introduce the following $\mathcal{T}$ and $\mathcal{C P} \mathcal{T}$ violation parameters:

$$
\begin{gathered}
\varepsilon_{\mathrm{T}} \equiv \sin \left(\varphi_{\mathrm{SW}}\right) \frac{\left|\Lambda_{12}\right|^{2}-\left|\Lambda_{21}\right|^{2}}{\Delta \Gamma \Delta m} \mathrm{e}^{i \varphi_{\mathrm{SW}}}, \\
\delta \equiv \cos \left(\varphi_{\mathrm{SW}}\right) \frac{\Lambda_{22}-\Lambda_{11}}{\Delta \Gamma} \mathrm{e}^{i\left(\varphi_{\mathrm{SW}}+\pi / 2\right)}
\end{gathered}
$$

with $\varphi_{\mathrm{SW}}=\operatorname{atan}(2 \Delta m / \Delta \Gamma)$. The lifetime difference is found to be about twice the mass difference $\Delta m \equiv m_{\mathrm{L}}-m_{\mathrm{S}}$ and therefore $\varphi_{\mathrm{SW}} \approx 45^{\circ}$. Assuming small $\mathcal{T}$ and $\mathcal{C P} \mathcal{T}$ violation, the time evolution of initially-pure strangeness states can be rewritten as

$$
\begin{aligned}
\left|\mathrm{K}^{0}(\tau)\right\rangle & =\left[f_{+}(\tau)-2 \delta f_{-}(\tau)\right]\left|\mathrm{K}^{0}\right\rangle+\left(1-2 \varepsilon_{\mathrm{T}}\right) \mathrm{e}^{-\mathrm{i} \varphi_{\Gamma}} f_{-}(\tau)\left|\overline{\mathrm{K}}^{0}\right\rangle, \\
\left|\overline{\mathrm{K}}^{0}(\tau)\right\rangle & =\left[f_{+}(\tau)+2 \delta f_{-}(\tau)\right]\left|\overline{\mathrm{K}}^{0}\right\rangle+\left(1+2 \varepsilon_{\mathrm{T}}\right) \mathrm{e}^{\mathrm{i} \varphi_{\Gamma}} f_{-}(\tau)\left|\mathrm{K}^{0}\right\rangle .
\end{aligned}
$$

Additional violations of discrete symmetries may occur in the decay of particles, either

1 through the interference of a decay amplitude with the oscillation amplitude, i.e. the phase of the decay amplitude is different from $\varphi_{\Gamma}$,

2 through the interference of two decay amplitudes with different weak phases, 3 or through direct $\mathcal{C P} \mathcal{T}$ in a decay amplitude.

The neutral kaon system is rather special compared to the other neutral meson systems $\left(D^{0}, B_{d}^{0}\right.$ and $\left.B_{s}^{0}\right)$, in the sense that due to the low mass of the kaon, the number of different final states is rather limited. This enables a rather complete 
systematic study of $\mathcal{C P}$ in the neutral kaon system. And in addition, one decay amplitude $\left(\mathrm{K}^{0} \rightarrow \pi \pi, I=0\right)$ dominates over all other decay amplitudes. This makes the effects of 1 and 2 very small in the kaon system while they are dominating in the $B$ systems.

In case, there is one amplitude contributing to the instant decay of a neutral kaon to a final state $f$, this can then be described for $\mathrm{K}^{0}$ and $\overline{\mathrm{K}}^{0}$ by the amplitudes $\mathcal{A}_{f}, \overline{\mathcal{A}}_{\bar{f}}$,

$$
\begin{aligned}
\mathcal{A}_{f} & =\left\langle f\left|\mathcal{H}_{\mathrm{wk}}\right| \mathrm{K}^{0}\right\rangle, \quad \overline{\mathcal{A}}_{\bar{f}}=\left\langle\bar{f}\left|\mathcal{H}_{\mathrm{wk}}\right| \overline{\mathrm{K}}^{0}\right\rangle, \\
& =\left(A_{f}+B_{f}\right) e^{i \delta_{f}}, \quad \overline{\mathcal{A}}_{\bar{f}}=\left(A_{f}^{*}-B_{f}^{*}\right) e^{i \delta_{f}} .
\end{aligned}
$$

The amplitudes $A_{f}$ and $B_{f}$ are $\mathcal{C P} \mathcal{T}$ symmetric and antisymmetric, respectively, $\delta_{f}$ is a strong phase describing a possible final state interaction. Alternatively we can express the rates in terms of $\mathrm{K}_{\mathrm{S}}$ and $\mathrm{K}_{\mathrm{L}}$ decay amplitudes, which is common in case $f$ is a $\mathcal{C P}$ eigenstate:

$$
\mathcal{A}_{f S}=\left\langle f\left|\mathcal{H}_{\mathrm{wk}}\right| \mathrm{K}_{\mathrm{S}}\right\rangle, \quad \mathcal{A}_{f L}=\left\langle f\left|\mathcal{H}_{\mathrm{wk}}\right| \mathrm{K}_{\mathrm{L}}\right\rangle
$$

It is then possible for example to calculate the time dependent decay rates into $f=\pi^{+} \pi^{-}$as:

$$
\begin{aligned}
& R_{\mathrm{K}^{0} \rightarrow \pi \pi}(\tau)=B\left[\mathrm{e}^{-\tau / \tau_{\mathrm{S}}}+\left|\eta_{+-}\right|{ }^{2} \mathrm{e}^{-\tau / \tau_{\mathrm{L}}}+2\left|\eta_{+-}\right| \mathrm{e}^{-\bar{\Gamma} \tau} \cos \left(\Delta m \tau-\phi_{+-}\right)\right], \\
& R_{\overline{\mathrm{K}}^{0} \rightarrow \pi \pi}(\tau)=\bar{B}\left[\mathrm{e}^{-\tau / \tau_{\mathrm{S}}}+\left|\eta_{+-}\right|^{2} \mathrm{e}^{-\tau / \tau_{\mathrm{L}}}-2\left|\eta_{+-}\right| \mathrm{e}^{-\bar{\Gamma} \tau} \cos \left(\Delta m \tau-\phi_{+-}\right)\right]
\end{aligned}
$$

with

$$
\eta_{+-}=\frac{\left\langle f\left|\mathcal{H}_{\mathrm{wk}}\right| \mathrm{K}_{\mathrm{L}}\right\rangle}{\left\langle f\left|\mathcal{H}_{\mathrm{wk}}\right| \mathrm{K}_{\mathrm{S}}\right\rangle}, \quad \bar{B} / B=\left[1+4 \Re\left(\varepsilon_{\mathrm{T}}+\delta\right)\right] .
$$

Since the $\pi^{+} \pi^{-}, \pi^{0} \pi^{0}$ final states are governed by isospin $I=0$ and $I=2$ amplitudes, with $\left|A_{2} / A_{0}\right| \approx 0.045,{ }^{26}$ the different contributions to $\eta_{+-}$and $\eta_{00}$ are given by:

$$
\begin{gathered}
\eta_{+-}=\varepsilon+\varepsilon^{\prime} \quad \text { and } \quad \eta_{00}=\varepsilon-2 \varepsilon^{\prime} \\
\text { with } \quad \varepsilon=\varepsilon_{\mathrm{T}}+\delta+i \Delta \phi+\Delta A
\end{gathered}
$$

where $\varepsilon_{\mathrm{T}}+\delta$ represent $\mathcal{T}$ and $\mathcal{C P} \mathcal{T}$ respectively in mixing, $\varepsilon^{\prime}$ direct $\mathcal{C P}$ through interference of $I=0$ and $I=2$ amplitudes, $\Delta \phi \mathcal{C P}$ through interference between mixing and $I=0$ decay amplitude, and $\Delta A$ represents $\mathcal{C P} \mathcal{T}$ in the dominating $I=0$ amplitude.

\subsection{Measurement of $\mathcal{C P}$ violation in the decay to $\pi^{+} \pi^{-}$}

The CPLEAR measurement of the decay rate asymmetry Fig. 2) shows that large rate differences between $\mathrm{K}^{0}$ and $\overline{\mathrm{K}}^{0}$ occur between 8 and $16 \mathrm{~K}_{\mathrm{S}}$ lifetimes, despite 


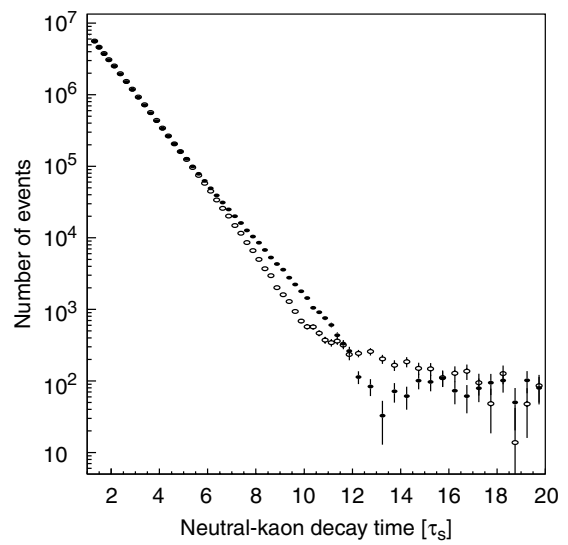

(a)

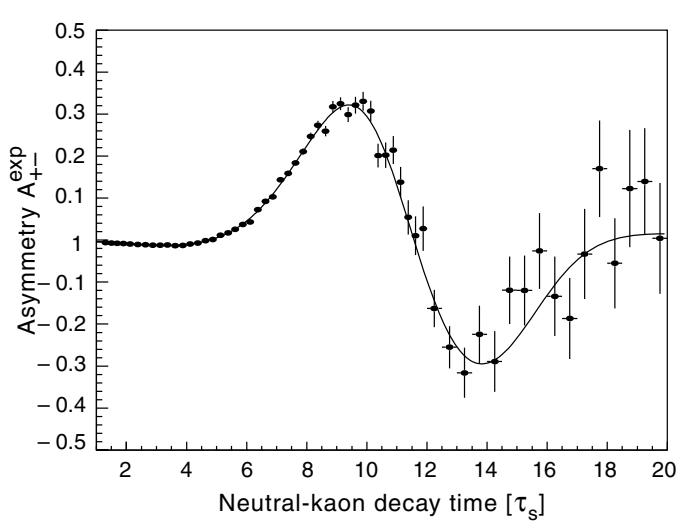

(b)

Fig. 2. Decay to $\pi^{+} \pi^{-}$: (a) The measured decay rate (acceptance corrected and background subtracted) as a function of the decay time $\tau$, separately for $\mathrm{K}^{0}$ (open circle) and $\overline{\mathrm{K}}^{0}$ (black circle). (b) The data points (black circle) are the measured time dependent decay-rate asymmetry $A_{+-}(\tau)$. The continuous curve is the result of the best fit (Eq. (23)).

the fact that $\left|\eta_{+-}\right|$is only about $\approx 2.3 \times 10^{-3}$. The measured decay rates need to be corrected for charge asymmetric detection efficiencies of the accompanying primary $\mathrm{K}^{ \pm} \pi^{\mp}$ pair, which is done using the high statistics data of the $\pi^{+} \pi^{-}$mode at short decay times, where contributions due to $\mathcal{C P}$ described by $\eta_{+-}$is known with sufficient accuracy. However, this only allows one to determine $w=\left[1+4 \Re\left(\varepsilon_{\mathrm{T}}+\delta\right)\right] \xi$, with $\xi$ describing the detector effects. The experimental measured asymmetry then becomes:

$$
\begin{aligned}
A^{+-}(\tau) & =\frac{R\left(\overline{\mathrm{K}}^{0} \rightarrow \pi^{+} \pi^{-}\right)-k * w R\left(\mathrm{~K}^{0} \rightarrow \pi^{+} \pi^{-}\right)}{R\left(\overline{\mathrm{K}}^{0} \rightarrow \pi^{+} \pi^{-}\right)+k * w R\left(\mathrm{~K}^{0} \rightarrow \pi^{+} \pi^{-}\right)} \\
& =-2 \frac{\left|\eta_{+-}\right| \mathrm{e}^{\frac{1}{2}\left(1 / \tau_{\mathrm{S}}-1 / \tau_{\mathrm{L}}\right) \tau} \cos \left(\Delta m \times \tau-\phi_{+-}\right)}{1+\left[\left|\eta_{+-}\right|^{2}+\operatorname{Bck}(\tau)\right] \mathrm{e}^{\left(1 / \tau_{\mathrm{S}}-1 / \tau_{\mathrm{L}}\right) \tau}}
\end{aligned}
$$

with $\operatorname{Bck}(\tau)$ describing the residual background contributions mainly from semileptonic decays, $k$ a free parameter of the fit accounting for the statistical uncertainties in the normalisation weights and for the correlations between the magnitudes of these weights and the fitted $\mathcal{C} \mathcal{P}$-violation parameters. Using the 1998 PDG average values for $\Delta m, \tau_{\mathrm{S}}$ and $\tau_{\mathrm{L}},{ }^{27}$ the final CPLEAR result is:

$$
\begin{aligned}
\left|\eta_{+-}\right| & =\left(2.264 \pm 0.023_{\text {stat }} \pm 0.026_{\text {syst }} \pm 0.007_{\tau_{\mathrm{S}}}\right) \times 10^{-3} \\
\phi_{+-} & =43.19^{\circ} \pm 0.53^{\circ}{ }_{\text {stat }} \pm 0.28^{\circ}{ }_{\text {syst }} \pm 0.42^{\circ}{ }_{\Delta m} .
\end{aligned}
$$

The improved precision in the value of the phase $\phi_{+-}$had been an important ingredient for setting a limit to a possible $\mathcal{C P} \mathcal{T}$ violating $\mathrm{K}^{0}-\overline{\mathrm{K}}^{0}$ mass difference, see Section 4.5. 


\subsection{Direct measurements of the $\mathcal{T}$ and $\mathcal{C} \mathcal{P} \mathcal{T}$ violation parameters}

Semileptonic decays of neutral kaons have the distinctive feature that the charge of the lepton tags the strangeness at the time of the decay $\left(\mathrm{K}^{0} \rightarrow \pi^{-} l^{+} \nu\right.$ and $\overline{\mathrm{K}}^{0} \rightarrow$ $\left.\pi^{0} l^{-} \bar{\nu}\right)$. Within the standard model, $\Delta S=\Delta Q$ violating decays $\left(\mathrm{K}^{0} \rightarrow \pi^{0} l^{-} \bar{\nu}\right.$ and $\left.\overline{\mathrm{K}}^{0} \rightarrow \pi^{-} l^{+} \nu\right)$ are expected to be heavily suppressed $\left(10^{-7}\right.$; Ref. 28$)$ and have not been observed so far, only upper limits have been measured. This allows one to measure for example, very precisely the oscillation frequency of an originally $\mathrm{K}^{0}$ state to change to a $\overline{\mathrm{K}}^{0}$ state, ${ }^{13}$ and moreover observe directly $\mathcal{T}$ violation by measuring the rate asymmetry between a $\mathrm{K}^{0}$ decaying as $\overline{\mathrm{K}}^{0}$ and its $\mathcal{T}$-conjugated process, $\overline{\mathrm{K}}^{0}$ decaying as $\mathrm{K}^{0} \cdot{ }^{10}$

In the absence of $\Delta S=\Delta Q$ violating processes, the time dependent decay rate asymmetry $A_{\mathrm{T}}$ measures directly the difference in magnitude of the off-diagonal elements of $\Lambda$ without any assumption about the smallness of $\mathcal{C P}$ and the magnitude of $\Delta \Gamma$ :

$$
A_{\mathrm{T}}(\tau)=\frac{R\left(\overline{\mathrm{K}}^{0} \rightarrow \mathrm{K}^{0}\right)(\tau)-R\left(\mathrm{~K}^{0} \rightarrow \overline{\mathrm{K}}^{0}\right)(\tau)}{R\left(\overline{\mathrm{K}}^{0} \rightarrow \mathrm{K}^{0}\right)(\tau)+R\left(\mathrm{~K}^{0} \rightarrow \overline{\mathrm{K}}^{0}\right)(\tau)}=\frac{\left|\Lambda_{12}\right|^{2}-\left|\Lambda_{21}\right|^{2}}{\left|\Lambda_{12}\right|^{2}+\left|\Lambda_{21}\right|^{2}}=4 \Re\left(\varepsilon_{\mathrm{T}}\right) .
$$

With $\Delta S=\Delta Q$ violating processes, three more parameters related to the semileptonic decay amplitudes appear in the formalism of semileptonic decay rate asymmetries: $\Re(y)$ describing direct $\mathcal{C P} \mathcal{T}$ violation in the $\Delta S=\Delta Q$ allowed decay, $\left(x_{+}\right) \mathcal{C P}$ violating and $\mathcal{C P} \mathcal{T}$ conserving and $\left(x_{-}\right) \mathcal{C P} \mathcal{T}$ violating contributions through $\Delta S=\Delta Q$ violating amplitudes. For a detailed definition see Ref. 1, Section 2.2. $A_{\mathrm{T}}$ then becomes:

$$
\begin{aligned}
A_{\mathrm{T}}(\tau) & =4 \Re\left(\varepsilon_{\mathrm{T}}\right)-2 \Re\left(x_{-}+y\right) \\
& +2 \frac{\Re\left(x_{-}\right)\left(\mathrm{e}^{-(1 / 2) \Delta \Gamma \tau}-\cos (\Delta m \tau)\right)+\Im\left(x_{+}\right) \sin (\Delta m \tau)}{\cosh \left(\frac{1}{2} \Delta \Gamma \tau\right)-\cos (\Delta m \tau)} \\
& \longrightarrow 4 \Re\left(\varepsilon_{\mathrm{T}}\right)-2 \Re\left(x_{-}+y\right) \quad \text { for } \tau \gg \tau_{\mathrm{S}}
\end{aligned}
$$

In addition, correcting for charge depending detector asymmetries affecting the detection of the accompanying primary particles $\left(\mathrm{K}^{ \pm} \pi^{\mp}\right)$ using the $\pi^{+} \pi^{-}$data at early lifetimes, yields an additional contribution to the asymmetry of $2 \Re\left(\varepsilon_{\mathrm{T}}+\delta\right)$. Using high precision measurements of the semileptonic decay asymmetry, ${ }^{27}$ $\delta_{l}=2 \Re\left(\varepsilon_{\mathrm{T}}+\delta-y-x_{-}\right)=(3.27 \pm 0.12) \times 10^{-3}$, this results in:

$$
\begin{aligned}
A_{\mathrm{T}}{ }^{\exp }(\tau) & =4 \Re\left(\varepsilon_{\mathrm{T}}\right)-\Re\left(x_{-}+y\right) \\
& +2 \frac{\Re\left(x_{-}\right)\left(\mathrm{e}^{-(1 / 2) \Delta \Gamma \tau}-\cos (\Delta m \tau)\right)+\Im\left(x_{+}\right) \sin (\Delta m \tau)}{\cosh \left(\frac{1}{2} \Delta \Gamma \tau\right)-\cos (\Delta m \tau)} .
\end{aligned}
$$




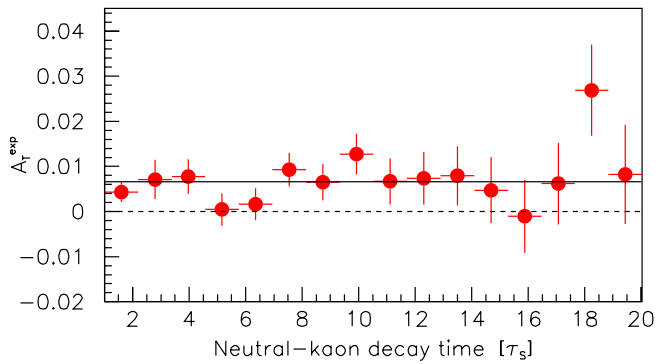

(a)

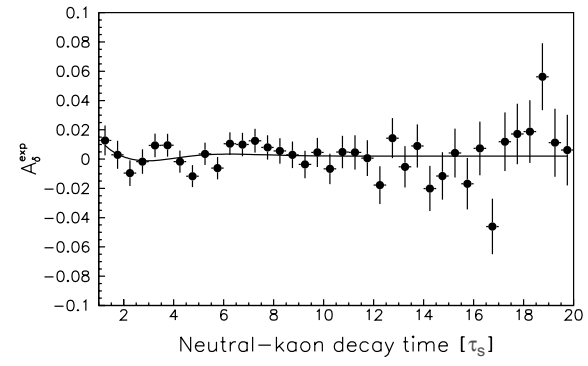

(b)

Fig. 3. (a) Experimental demonstration of T-violation: the asymmetry $A_{T}^{\exp }$ versus the neutralkaon decay time (in units of $\tau_{\mathrm{S}}$ ). The positive values show that a $\overline{\mathrm{K}}^{0}$ develops into a $\mathrm{K}^{0}$ with higher probability than does a $\mathrm{K}^{0}$ into a $\overline{\mathrm{K}}^{0}$. The solid line represents the fitted average $\left\langle A_{T}^{\exp }\right\rangle=$ $(6.6 \pm 1.3) \times 10^{-3}$. (b) The experimentally measured $\mathcal{C P} \mathcal{T}$ violating asymmetry $A_{\delta}$. The solid line represents the result of the fit.

In the original publication of the $A_{\mathrm{T}}$ asymmetry, ${ }^{10}$ an assumption about $\mathcal{C P} \mathcal{T}$ invariance in the semileptonic decay amplitudes was made when fitting the experimental data (Fig. 3) resulting in:

$$
\begin{gathered}
4 \Re\left(\varepsilon_{\mathrm{T}}\right)=\left(6.2 \pm 1.4_{\text {stat }} \pm 1.0_{\text {syst }}\right) \times 10^{-3}, \\
\Im\left(x_{+}\right)=\left(1.2 \pm 1.9_{\text {stat }} \pm 0.9_{\text {syst }}\right) \times 10^{-3}
\end{gathered}
$$

having observed directly for the first time $\mathcal{T}$ violation at work. Compiling the CPLEAR data together with other world averages for some of the neutral kaon parameters, together with the Bell-Steinberger (or unitarity) relation, ${ }^{20}$ constraints the quantity $\Re\left(x_{-}+y\right)$ to be within $(-0.2 \pm 0.3) \times 10^{-3}$ confirming the assumption that the possible contribution to $A_{\mathrm{T}} \exp$ from $\mathcal{C P} \mathcal{T}$-violating decay amplitudes is negligible. Until today (2014), this is the only direct observation of $\mathcal{T}$ violation in the mixing of neutral mesons.

A similar asymmetry can be constructed for the case of $\mathcal{C P} \mathcal{T}$, for simplicity assume absence of $\Delta S=\Delta Q$ violating processes:

$$
\begin{aligned}
A_{\mathrm{CPT}}(\tau) & =\frac{R\left(\overline{\mathrm{K}}^{0} \rightarrow \overline{\mathrm{K}}^{0}\right)(\tau)-R\left(\mathrm{~K}^{0} \rightarrow \mathrm{K}^{0}\right)(\tau)}{R\left(\overline{\mathrm{K}}^{0} \rightarrow \overline{\mathrm{K}}^{0}\right)(\tau)+R\left(\mathrm{~K}^{0} \rightarrow \mathrm{K}^{0}\right)(\tau)} \\
& =2 \Re(y)+4 \frac{\Re(\delta) \sinh \left(\frac{1}{2} \Delta \Gamma \tau\right)+\Im(\delta) \sin (\Delta m \tau)}{\cosh \left(\frac{1}{2} \Delta \Gamma \tau\right)+\cos (\Delta m \tau)} .
\end{aligned}
$$

Including $\Delta S=\Delta Q$ violating contributions and correcting for the primary charge asymmetry as before with the $2 \pi$ data, if finally turns out that a direct measurement of $\mathcal{C P} \mathcal{T}$ can be obtained by combining the two asymmetries 26 and 
29 to become:

$$
\begin{aligned}
A_{\delta}(\tau)= & A_{\mathrm{CPT}}^{\exp }(\tau)+A_{\mathrm{T}}{ }^{\exp }(\tau) \\
= & 4 \Re(\delta)+4 \frac{\Re(\delta) \sinh \left(\frac{1}{2} \Delta \Gamma \tau\right)+\Im(\delta) \sin (\Delta m \tau)}{\cosh \left(\frac{1}{2} \Delta \Gamma \tau\right)+\cos (\Delta m \tau)} \\
& -4 \frac{\Re\left(x_{-}\right) \cos (\Delta m \tau) \sinh \left(\frac{1}{2} \Delta \Gamma \tau\right)-\Im\left(x_{+}\right) \sin (\Delta m \tau) \cosh \left(\frac{1}{2} \Delta \Gamma \tau\right)}{\left[\cosh \left(\frac{1}{2} \Delta \Gamma \tau\right)\right]^{2}-[\cos (\Delta m \tau)]^{2}} \\
\longrightarrow & 8 \Re(\delta) \quad \text { for } \tau \gg \tau_{\mathrm{S}} .
\end{aligned}
$$

The final fit results are:

$$
\begin{gathered}
\Re(\delta)=\left(3.0 \pm 3.3_{\text {stat }} \pm 0.6_{\text {sys }}\right) \times 10^{-4}, \\
\Im(\delta)=\left(-1.5 \pm 2.3_{\text {stat }} \pm 0.3_{\text {sys }}\right) \times 10^{-2}, \\
\Re\left(x_{-}\right)=\left(0.2 \pm 1.3_{\text {stat }} \pm 0.3_{\text {sys }}\right) \times 10^{-2}, \\
\Im\left(x_{+}\right)=\left(1.2 \pm 2.2_{\text {stat }} \pm 0.3_{\text {sys }}\right) \times 10^{-2} .
\end{gathered}
$$

\section{5. $\mathcal{T}$ and $\mathcal{C P} \mathcal{T}$ parameters constrained by the unitarity relation}

As mentioned earlier, the neutral kaon system is unique in the sense that due to the rather limited number of final states, Eq. (5) can directly be used as a constraint by summing up all relevant final states. By improving the precision of the three-pion decay rates ${ }^{8,9}$ and measuring precisely the semileptonic decay rates, ${ }^{12}$ CPLEAR made possible the determination of many parameters of the neutral kaon system with unprecedented accuracy. Rewriting Eq. (5) in the $\mathrm{K}_{\mathrm{S}}-\mathrm{K}_{\mathrm{L}}$ basis, we derive the well known Bell-Steinberger relation ${ }^{29,} 30$ relating all decay channels of neutral kaons to the parameters describing $\mathcal{T}$ and $\mathcal{C P} \mathcal{T}$ non-invariance in the neutral kaon mixing:

$$
\Re\left(\varepsilon_{\mathrm{T}}\right)-i \Im(\delta)=\frac{1}{2(i \Delta m+\bar{\Gamma})} \times \sum\left\langle f\left|\mathcal{H}_{\mathrm{wk}}\right| \mathrm{K}_{\mathrm{L}}\right\rangle^{*}\left\langle f\left|\mathcal{H}_{\mathrm{wk}}\right| \mathrm{K}_{\mathrm{S}}\right\rangle
$$

The sum on the right-hand side of the above equation can be written as

$$
\begin{aligned}
\sum\left\langle f\left|\mathcal{H}_{\mathrm{wk}}\right| \mathrm{K}_{\mathrm{L}}\right\rangle^{*}\left\langle f\left|\mathcal{H}_{\mathrm{wk}}\right| \mathrm{K}_{\mathrm{S}}\right\rangle= & \sum\left(\mathrm{BR}_{\pi \pi}^{\mathrm{S}} \Gamma_{\mathrm{S}} \eta_{\pi \pi}\right)+\sum\left(\mathrm{BR}_{\pi \pi \pi}^{\mathrm{L}} \Gamma_{\mathrm{L}} \eta_{\pi \pi \pi}^{*}\right) \\
& +2\left[\Re\left(\varepsilon_{\mathrm{T}}\right)-\Re(y)-i\left(\Im\left(x_{+}\right)+\Im(\delta)\right)\right] \mathrm{BR}_{1 \pi \nu}^{\mathrm{L}} \Gamma_{\mathrm{L}} .
\end{aligned}
$$

Here BR stands for branching ratio, the upper index refers to the decaying particle and the lower index to the final state and $l$ denotes electrons and muons. The radiative modes, like $\pi^{+} \pi^{-} \gamma$, are essentially included in the corresponding parent modes. Channels with $\mathrm{BR}_{f}^{\mathrm{S}}\left(\right.$ or $\left.\mathrm{BR}_{f}^{\mathrm{L}} \times \Gamma_{L} / \Gamma_{S}\right)<10^{-5}$ do not contribute to Eq. (31) within the accuracy of the CPLEAR analysis. Using data from CPLEAR together 
with the most recent world averages (1998) for some of the neutral kaon parameters, the following result is being obtained: ${ }^{20}$

$$
\begin{gathered}
\Re\left(\varepsilon_{\mathrm{T}}\right)=\left(164.9 \pm 2.52_{\text {stat }} \pm 0.1_{\text {sys }}\right) \times 10^{-5}, \\
\Im(\delta)=\left(2.4 \pm 5.02_{\text {stat }} \pm 0.1_{\text {sys }}\right) \times 10^{-5}, \\
\Re(\delta)=\left(2.4 \pm 2.72_{\text {stat }} \pm 0.6_{\text {sys }}\right) \times 10^{-4},
\end{gathered}
$$

which establishes unambiguously $\mathcal{T}$ violation at the level of $65 \sigma$ and sets stringent limits on $\mathcal{C P} \mathcal{T}$ in mixing but also in various decay amplitudes, for more results see Ref. 20. The unitarity relation in the $\mathrm{K}^{0}-\overline{\mathrm{K}}^{0}$ basis can also be used to derive a limit on the phase difference between $\Gamma_{12}$ and the dominating $I=0$ decay amplitude in the $\pi \pi$ mode. In the neutral $B$-system, such a phase difference which corresponds to the interference of mixing and decay amplitudes is the dominating source for $\mathcal{C P}$ violation. In the kaon system, it is small $\Delta \Phi=\frac{1}{2}\left[\varphi_{\gamma}-\arg \left(A_{0}^{*} \bar{A}_{0}\right)\right]=(-1.2 \pm 8.5) \times$ $10^{-6} .31$

The $\mathcal{C P} \mathcal{T}$ theorem, ${ }^{32-34}$ which is based on general principles of the relativistic quantum field theory, states that any order of the triple product of the discrete symmetries $\mathcal{C}, \mathcal{P}$ and $\mathcal{T}$ should represent an exact symmetry. The theorem predicts, among other things, that particles and antiparticles have equal masses and lifetimes. With the above results for $\Re(\delta)$ and $\Im(\delta)$ and using Eq. (15), it is straightforward to obtain:

$$
\begin{aligned}
& \Gamma_{\mathrm{K}^{0} \mathrm{~K}^{0}}-\Gamma_{\overline{\mathrm{K}}^{0} \overline{\mathrm{K}}^{0}}=(3.9 \pm 4.2) \times 10^{-18} \mathrm{GeV}, \\
& \mathrm{M}_{\mathrm{K}^{0} \mathrm{~K}^{0}}-\mathrm{M}_{\overline{\mathrm{K}}^{0} \overline{\mathrm{K}}^{0}}=(-1.5 \pm 2.0) \times 10^{-18} \mathrm{GeV},
\end{aligned}
$$

with a correlation coefficient of -0.95 . In contrast to earlier compilations, for example Ref. 27, the CPLEAR results are free of any prejudice of $\mathcal{C P} \mathcal{T}$ invariance in decay amplitudes. Assuming $\mathcal{C P} \mathcal{T}$ invariance in all decays, the precision on the mass difference $(-0.7 \pm 2.8) \times 10^{-19} \mathrm{GeV}$ improves by about one order of magnitude. These are still the best limits for a mass difference between particles and antiparticles, thanks to the small value of $\Delta m=3.484 \times 10^{-12} \mathrm{MeV}$ which works like a magnification glass. In the neutral $B$-systems, the mass differences are $\approx 100$ and $\approx 300$ larger for the $B_{d}$ and $B_{s}$ respectively compared to the kaon system and therefore $B$-systems are less sensitive to $\mathcal{C P} \mathcal{T}$ effects.

\subsection{Measurements related to basic principles}

In the last section, I would like to discuss three CPLEAR results ${ }^{16,}$ 18, 19 which are related to the basics of Quantum Mechanics (QM) and general relativity.

\subsubsection{Probing a possible loss of $Q M$ coherence}

All results discussed so far are based on a framework of QM of closed systems, solutions of Eq. (4) are pure states and evolve as such in time. Some approaches 
to quantum gravity ${ }^{35}$ suggest that topologically non-trivial space-time fluctuations (space-time foam, virtual black-holes) entail an intrinsic, fundamental information loss, and therefore induce transitions from pure to mixed states, ${ }^{36}$ and define the arrow of time. In the $\mathrm{K}^{0}-\overline{\mathrm{K}}^{0}$ system such a behavior can be described by a phenomenological ansatz using a $2 \times 2$ density matrix $\rho$, which obeys

$$
\dot{\rho}=-i\left[\Lambda \rho-\rho \Lambda^{\dagger}\right]+\delta / \Lambda \rho
$$

where $\Lambda$ is the $2 \times 2$ matrix of Eq. (6), and the term $\not \Lambda \rho$ induces a loss of quantum coherence in the observed system. In the case of the neutral kaon system, if the conservation of energy and strangeness are assumed, the open-system equation (33) introduces $^{36}$ three $\mathcal{C P} \mathcal{T}$-violation parameters $\alpha, \beta$ and $\gamma$. Before CPLEAR, existing measurements of $\mathcal{C P}$ violation in the mixing of neutral kaons could have been solely explained by these $\mathcal{C P} \mathcal{T}$-violation parameters. Having measured decay-rate asymmetries over a large range of lifetimes $\left(\sim 20 \tau_{\mathrm{S}}\right)$ for the $\pi^{+} \pi^{-}$and e $\pi \nu$ decay channels together with the constraint of $\left|\eta_{+-}\right|$and $\delta_{l}$ measured at long lifetimes, ${ }^{27}$ enabled CPLEAR to obtain 90\% CL limits, $\alpha<4.0 \times 10^{-17} \mathrm{GeV}, \beta<2.3 \times$ $10^{-19} \mathrm{GeV}$ and $\gamma<3.7 \times 10^{-21} \mathrm{GeV}$, to be compared with a possible order of magnitude of $\mathcal{O}\left(m_{\mathrm{K}}^{2} / m_{\text {Planck }}\right)=2 \times 10^{-20} \mathrm{GeV}$ for such effects if relevant for our universe.

\subsubsection{Testing the non-separability of the $\mathrm{K}^{0} \overline{\mathrm{K}}^{0}$ wave function}

For this measurement, pairs of $\mathrm{K}^{0} \overline{\mathrm{K}}^{0}$ were selected being produced simultaneously in the reaction:

$$
\overline{\mathrm{p}} \mathrm{p} \rightarrow \mathrm{K}^{0} \overline{\mathrm{K}}^{0} .
$$

Depending on the angular momentum between the $\mathrm{K}^{0}$ and $\overline{\mathrm{K}}^{0}$, the wave function describing the time evolution of the two entangled states is either symmetric or antisymmetric with respect of changing $\mathrm{K}^{0} \leftrightarrow \overline{\mathrm{K}}^{0}$. However, it turns out that in $93 \%$ of the cases, ${ }^{37}$ the wave function is antisymmetric with $J^{P C}=1^{--}$:

$$
\langle\Psi(0,0)|=\frac{1}{\sqrt{2}}\left[\left\langle\mathrm { K } ^ { 0 } | _ { a } \left\langle\left.\overline{\mathrm{K}}^{0}\right|_{b}-\left\langle\left.\overline{\mathrm{K}}^{0}\right|_{a}\left\langle\left.\mathrm{~K}^{0}\right|_{b}\right]\right.\right.\right.\right.
$$

Switching on the time evolution of the neutral kaons, Eq. (8), and separating into combinations of unlike and like-strangeness at time $t_{a}$ and $t_{b}$ yields:

$$
\begin{aligned}
& \left\langle\left.\Psi\left(t_{a}, t_{b}\right)\right|_{\mathrm{K}^{0} \overline{\mathrm{K}}^{0}} \propto T_{11}\left(t_{a}\right) T_{22}\left(t_{b}\right)-T_{12}\left(t_{a}\right) T_{21}\left(t_{b}\right),\right. \\
& \left\langle\left.\Psi\left(t_{a}, t_{b}\right)\right|_{\overline{\mathrm{K}}^{0} \overline{\mathrm{K}}^{0}} \propto T_{21}\left(t_{a}\right) T_{22}\left(t_{b}\right)-T_{22}\left(t_{a}\right) T_{21}\left(t_{b}\right) .\right.
\end{aligned}
$$

From which follows the prediction of QM, independent of any $\mathcal{C P}$ in the mixing of neutral kaons: at equal times, the probability to observe the two states with equal strangeness goes to zero. 


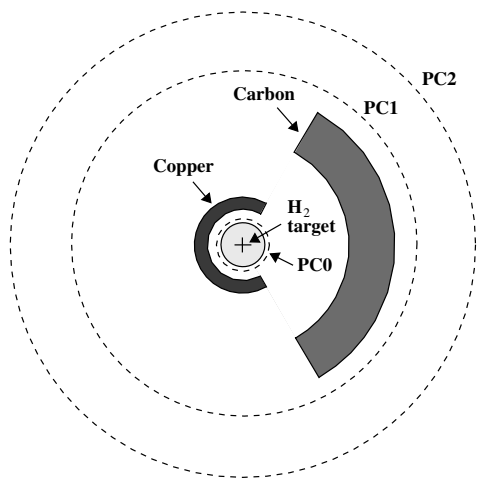

(a)

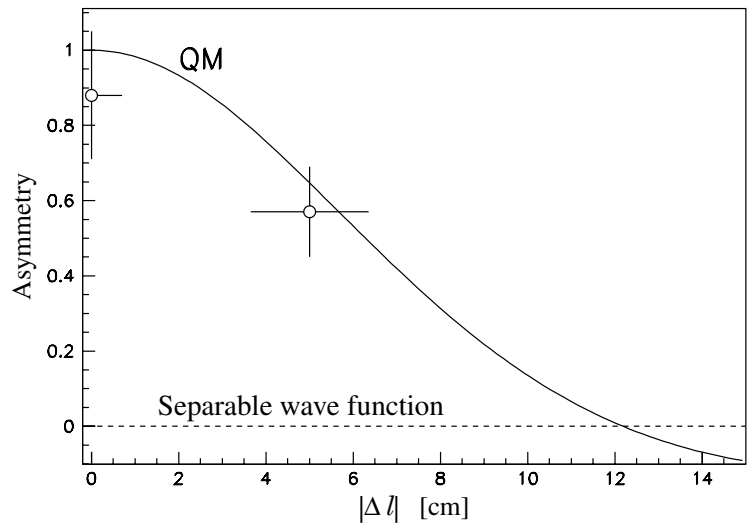

(b)

Fig. 4. (a) Conceptual sketch of the experiment (see text); (b) asymmetry of the measured $\Lambda \mathrm{K}^{ \pm}$ yields after background subtraction. The two points show the long distance correlation of the entangled kaons, in agreement with quantum mechanics.

The speciality of this measurement, the strangeness is monitored by strong interaction in two absorbers near the target, see Fig. 4, via the observation in the same event, at two different times, of a $\Lambda$ and a $\mathrm{K}^{+}$(unlike strangeness) or a $\Lambda$ and a $\mathrm{K}^{-}$or two $\Lambda$ (like strangeness). The tagging via strong interaction bypasses any potential complications arising from $\Delta S=\Delta Q$ violating neutral meson decays. The asymmetries of the yields for unlike- and like-strangeness events were measured for two experimental configurations $\mathrm{C}(0)$ and $\mathrm{C}(5)$, see Fig. 4(a), corresponding to $\approx 0$ and $1.2 \tau_{\mathrm{S}}$ proper time differences between the two strangeness measurements, or path differences $|\Delta l|$ of $\approx 0$ and $5 \mathrm{~cm}$. As shown in Fig. 4(b), these asymmetries are consistent with the values predicted from QM, and therefore consistent with the non-separability hypothesis of the $\mathrm{K}^{0} \overline{\mathrm{K}}^{0}$ wave function. The non-separability hypothesis is also strongly favoured by the yield of $\Lambda \Lambda$ events.

\subsubsection{Test of the equivalence principle for particles and antiparticles}

With the large statistics of $\pi^{+} \pi^{-}$decays, CPLEAR had been able to search for possible annual, monthly and diurnal modulations of the observables $\left|\eta_{+-}\right|$and $\phi_{+-}$that could be correlated with variations in astrophysical potentials. No such correlations were found within the CPLEAR accuracy. ${ }^{16}$ Data were analyzed assuming effective scalar, vector and tensor interactions, with the conclusion that the principle of equivalence between particles and antiparticles holds to a level of $(6.5,4.3,1.8) \times 10^{-9}$, respectively, for scalar, vector and tensor potential originating from the Sun with a range much greater than the Earth-Sun distance. Figure 5 shows a compilation of the upper limits on $|g-\bar{g}|_{J}$, the gravitational coupling difference between $\mathrm{K}^{0}$ and $\overline{\mathrm{K}}^{0}$, as a function of the interaction range $r_{J}$ where $J=0,1,2$ for scalar, vector and tensor potential, respectively. 


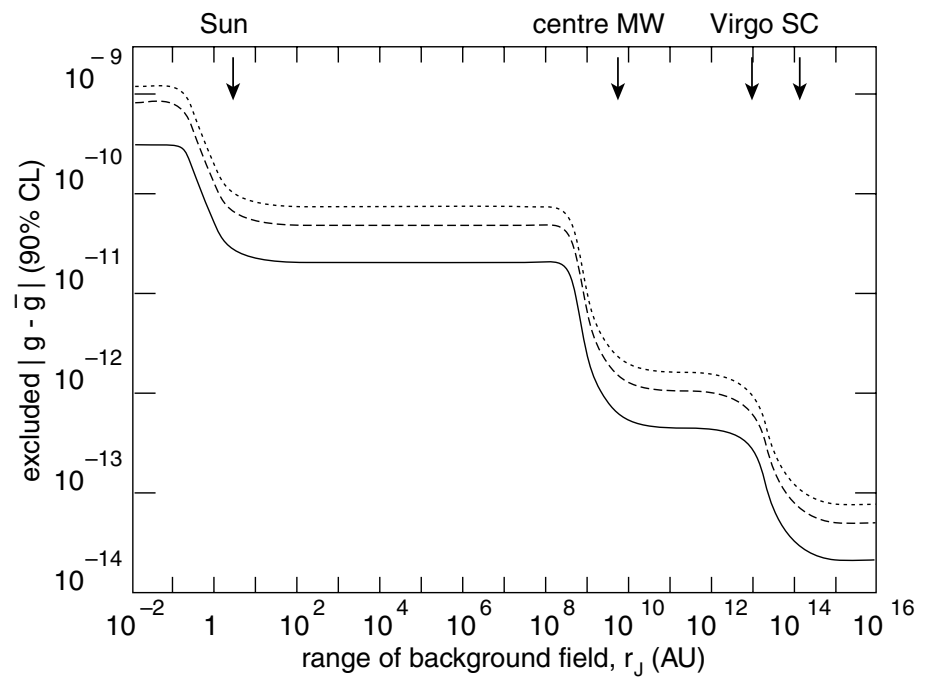

Fig. 5. Limits on the gravitational coupling difference between $\mathrm{K}^{0}$ and $\overline{\mathrm{K}}^{0},|g-\bar{g}|_{J}$, obtained from the measured $\mathrm{K}^{0}-\overline{\mathrm{K}}^{0}$ mass difference as a function of the effective interaction range $r_{J}$, with $J=0,1,2$ for scalar, vector and tensor potential, respectively. Labels along the top indicate the distances to several astronomical bodies (Milky Way: MW; Shapley supercluster: SC) measured in Astronomical Units (AU). The curves are upper limits shown separately for tensor (solid line), vector (dashed line) and scalar (dotted line) interactions.

\section{Conclusion}

To summarise, CPLEAR had been a nice small size experiment studying with unprecedented precision violations of discrete symmetries $(\mathcal{T}, \mathcal{C P} \mathcal{T}$ and $\mathcal{C P})$ in the neutral kaon systems and addressing fundamental physics questions ranging from a possible breakdown of quantum coherence of the wave function to the equivalence principle of general relativity. Thanks to the idea of using flavour tagged neutral kaon "beams".

\section{References}

1. A. Angelopoulos et al., Physics at CPLEAR, Physics Reports 374, 165-270 (2003).

2. S. Baird et al., in Proc of the 1997 Particle Accelerator Conference, Vancouver, M. Comyn et al. (ed.) (IEEE, Piscataway, 1998), p. 982.

3. CERN, (2014). https://home.web.cern.ch/about/accelerators/low-energy-antiprotonring.

4. A. Angelopoulos et al., Measurement of the neutral kaon regeneration amplitude in carbon at momenta below 1-GeV/c, Phys. Lett. B 413, 422 (1997).

5. R. Adler et al., Nucl. Instrum. Methods A 379, 76 (1996).

6. A. Apostolakis et al., A detailed description of the analysis of the decay of neutral kaons to $\pi^{+} \pi^{-}$in the cplear experiment, Eur. Phys. J. C 18, 41 (2000). 
7. A. Angelopoulos et al., Measurement of the $\mathrm{CP}$ violation parameter $\mathrm{n}_{00}$ using tagged $\overline{\mathrm{K}}^{0}$ and $\mathrm{K}^{0}$, Phys. Lett. B 420, 191 (1998).

8. A. Angelopoulos et al., The neutral kaons decays to $\pi^{+} \pi^{-} \pi^{0}$ : a detailed analysis of the CPLEAR data, Eur. Phys. J. C 5, 389 (1998).

9. A. Angelopoulos et al., Search for $\mathrm{CP}$ violation in the decay of tagged $\overline{\mathrm{K}}^{0}$ and $\mathrm{K}^{0}$ to $\pi^{0} \pi^{0} \pi^{0}$, Phys. Lett. B 425, 391 (1998).

10. A. Angelopoulos et al., First direct observation of time-reversal non-invariance in the neutral-kaon system, Phys. Lett. 444, 43 (1998).

11. A. Angelopoulos et al., A determination of the CPT violation parameter $\operatorname{Re}(\delta)$ from the semileptonic decay of strangeness-tagged neutral kaons, Phys. Lett. 444, 52 (1998).

12. A. Angelopoulos et al., T-violation and CPT-invariance measurements in the CPLEAR experiment: a detailed description of the analysis of neutral-kaon decays to e $\pi \nu$, Eur. Phys. J. C 22, 55 (2001).

13. A. Angelopoulos et al., Measurement of the $\mathrm{K}_{\mathrm{L}}-\mathrm{K}_{\mathrm{S}}$ mass difference using semileptonic decays of tagged neutral kaons, Phys. Lett. 444, 38 (1998).

14. A. Apostolakis et al., Measurement of the energy dependence of the form factor $\mathrm{f}_{+}$ in $\mathrm{K}_{\mathrm{e} 3}^{0}$ decay, Phys. Lett. 473, 186 (2000).

15. A. Apostolakis et al., Measurement of the energy dependence of the form factor $\mathrm{f}_{+}$ in $\mathrm{K}_{\mathrm{e} 3}^{0}$ decay, Phys. Lett. 473, 186 (2000).

16. A. Apostolakis et al., Tests of the equivalence principle with neutral kaons, Phys. Lett. 452, 425 (1999).

17. W. Fetscher et al., Regeneration of arbitrary coherent neutral kaon states: A new method for measuring the $\mathrm{K}^{0}-\overline{\mathrm{K}}^{0}$ forward scattering amplitude, Z. Phys. C 72, 543 (1996).

18. A. Apostolakis et al., An epr experiment testing the non-separability of the $\overline{\mathrm{K}}^{0}$ $\mathrm{K}^{0}$ wave function, Phys. Lett. B. 422, 339 (1998).

19. A. Angelopoulos et al., Test of CPT symmetry and quantum mechanics with experimental data from CPLEAR, Phys. Lett. 364, 239 (1995).

20. A. Apostolakis et al., Determination of the $\mathrm{T}$ and CPT violation parameters in the neutral kaon system using the Bell-Steinberger relation and data from CPLEAR, Phys. Lett. B 456, 297-303 (1999). doi: 10.1016/S0370-2693(99)00483-9.

21. A. Angelopoulos et al., $\mathrm{K}^{0}-\overline{\mathrm{K}}^{0}$ mass and decay-width differences: CPLEAR evaluation, Phys. Lett. 471, 332 (1999).

22. V. Weisskopf and E. Wigner, Over the natural line width in the radiation of the harmonius oscillator, Z. Phys. 65, 18-29 (1930). doi: 10.1007/BF01397406.

23. V. Weisskopf and E. P. Wigner, Calculation of the natural brightness of spectral lines on the basis of Dirac's theory, Z. Phys. 63, 54-73 (1930). doi: 10.1007/BF01336768.

24. S. Treiman and R. Sachs, Alternate modes of decay of neutral K mesons, Phys.Rev. 103, 1545-1549 (1956). doi: 10.1103/PhysRev.103.1545.

25. T. Lee, R. Oehme, and C.-N. Yang, Remarks on possible noninvariance under time reversal and charge conjugation, Phys. Rev. 106, 340-345 (1957). doi: 10.1103/ PhysRev.106.340.

26. T. J. Devlin and J. O. Dickey, Weak hadronic decays: $K \rightarrow 2 \pi$ and $K \rightarrow 3 \pi$, Rev. Mod. Phys. 51, 237 (1979). doi: 10.1103/RevModPhys.51.237.

27. C. Caso et al., Review of particle physics, Phys. J. C 3, 1 (1998). 
28. C. Dib and B. Guberina, Almost forbidden $\Delta \mathrm{Q}=-\Delta \mathrm{S}$ processes, Phys. Lett. $B$ 255, 113-116 (1991). doi: 10.1016/0370-2693(91)91149-P.

29. J. S. Bell and J. Steinberger, Weak interactions of kaons, in Proc. of the Oxford International Conference on Elementary Particles, R. G. Moorhouse et al. (eds.), (Oxford University Press, 1966), p. 195.

30. K. R. Schubert, B. Wolff, J. Chollet, J. Gaillard, M. Jane, et al., The phase of $\eta_{00}$ and the invariances CPT and T, Phys. Lett. B 31, 662-665 (1970). doi: 10.1016/03702693(70)90029-8.

31. T. Ruf, Status of CP and CPT violation in the neutral kaon system, in Proc of the 16th International Conference on Physics in Collision, Mexico City, Mexico, 19-21 June 1996 (1996).

32. R. S. B. House, ed. Time Reversal in Field Theory, Vol. 231, (1955). doi: 10.1098/ rspa.1955.0189.

33. R. Jost, A remark on the C.T.P. theorem, Helv. Phys. Acta. 30, 409-416 (1957).

34. G. Luders, Proof of the TCP theorem, Annals of Physics 2, 1-15 (1957). doi: 10.1016/ 0003-4916(57)90032-5.

35. S. Hawking, The unpredictability of quantum gravity, Commun. Math. Phys. 87, 395-415 (1982). doi: 10.1007/BF01206031.

36. J. R. Ellis, J. Hagelin, D. V. Nanopoulos, and M. Srednicki, Search for violations of quantum mechanics, Nucl. Phys. B 241, 381 (1984). doi: 10.1016/0550-3213(84) 90053-1.

37. R. Adler et al., Experimental measurement of the $K_{S} K_{S} / K_{S} K_{L}$ ratio in antiproton annihilations at rest in gaseous hydrogen at 15 and 27 bar, Phys. Lett. B 403, 383-389 (1997). doi: 10.1016/S0370-2693(97)00489-9. 